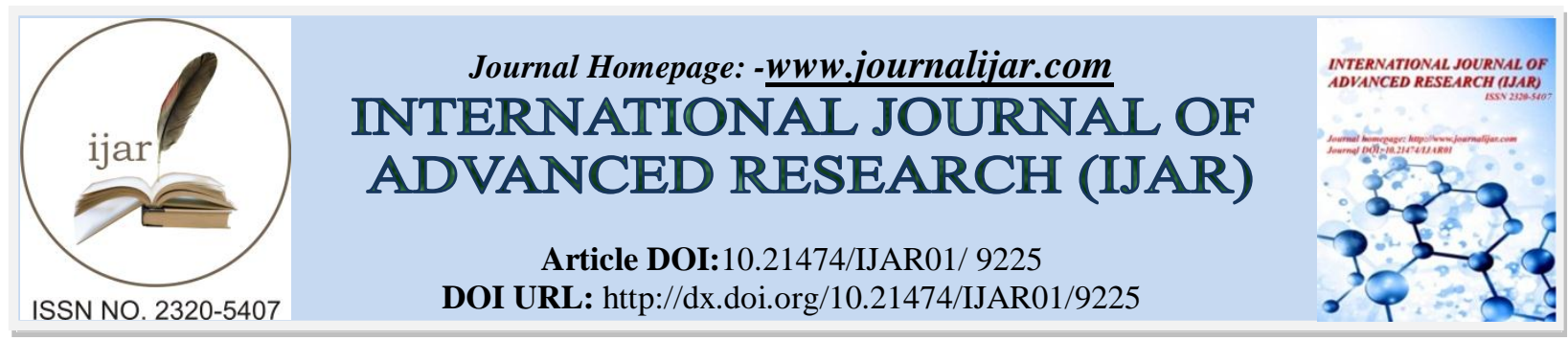

RESEARCH ARTICLE

\title{
LARGE LEFT VENTRICULAR INFEROBASAL ANEURYSM FOLLOWING CIRCUMFLEX ARTERY OCCLUSION.
}

\author{
Echerki Sara $^{1}$, Kaddaf Sofia ${ }^{1}$, Manouri Kawtar ${ }^{1}$ and Abelmajid Bouzerda ${ }^{1}$
}

Department of Cardiology, Mohammed V military hospital, Rabat, Morocco.

\section{Manuscript Info}

Manuscript History

Received: 06 April 2019

Final Accepted: 08 May 2019

Published: June 2019

\section{Abstract}

The majority of cardiac left ventricular aneurysms involve the anterior and/or apical wall. We present a case of a 57-year-old man with acute coronary syndrome with ST segment elevation complicated of a large inferobasal left ventricular aneurysm which remains rare.

Key words:-

True ventricular aneurysm, severe mitral

regurgitation, coronary angiography,

ventriculography, echocardiography.

Copy Right, IJAR, 2019,. All rights reserved.

\section{Introduction:-}

Left ventricular aneurysms are mechanic complications of myocardial infarction (MI). They are hemodynamically important pathologies. They were developing frequently during post-myocardial infarction period before reperfusion era; fortunately nowadays they are seen very rarely. We report the case of a patient who presented with inferior myocardial infarction that caused development of a rare aneurysm in the basal segment of the inferior wall, and summarize the literature regarding diagnosis, prognosis and clinical history of this particular finding.

\section{Case report :}

We report the case of 57 year old man without medical history, admitted to our cardiology department for acute coronary syndrome with ST segment elevation. The electrocardiogram showed ST - segment elevation with Q waves in the inferior leads. Transthoracic echocardiography revealed a large diskentic aneurysm involving the inferobasal wall of the left ventricule, measuring $40 * 30 \mathrm{~mm}$ without thrombus. The LV was not dilated with normal ejection fraction ( EF) of $65 \%$. The coronary angiography showed an occlusion of the circumflex coronary artery. Medical therapy was prescribed instead of stent implantation .

\section{Discussion:-}

Left ventricular aneurysm consists of localized area of myocardium with abnormal outward bulging and deformation during systole and diastole. The aneurysms appear like a thin, akinetic or dyskinetic region and collapse inward when the ventricle is vented during surgery. Aneurysm formation is a consequence of infarct expansion (1).

Clinical data suggest that infarct expansion occurs in approximately $35 \%$ to $45 \%$ of anterior MI and to a lesser extent in infarctions at other sites (1).Basal inferior wall aneurysms constitute nearly $3 \%$ of all LV aneurysms (2) The incidence of LV aneurysm has decreased from about $18,8 \%$ to $7,2 \%$ in recent years due to improvement in the management of acute coronary syndromes, including thrombolysis and angioplasty (3).

Aneurysms are seen four times more often as a complication of anterior MI than inferior MI (4). Besides atherosclerotic coronary artery disease, other uncommon causes of LV aneurysm include hypertrophic 
cardiomyopathy, arrhythmogenic right ventricular dysplasia, myocarditis, coronary artery fistula, chest trauma, infections like Chaga's disease and HIV, and connective tissue diseases like sarcoidosis and systemic lupus (5).

Transthoracic and transesophageal echocardiography are the most useful modalities used in the evaluation of left ventricular aneurysm because of their reliability and reproducibility.

Cardiac magnetic resonance imaging and ventriculography can provide additional information, particularly in the differential diagnosis of pseudoaneurysm. The finding of an inferobasal LV aneurysm requires an accurate differential diagnosis of pseudoaneurysm that typically can develop in posterior-inferior segments.

Histopathologically, the pseudoaneurysm wall consists of only the pericardial layer while a true aneurysm is delimited by all three layers (pericardium, myocardium, endocardium). The presence of a turbulent flow at the neck by color Doppler has been proposed as sign of pseudoaneurysm, but the absence of turbulence cannot exclude this finding $(6,7)$. Stagnant flow inside the aneurysm can lead to formation of thrombus and consequent embolism, therefore an accurate evaluation of the internal surface of the cavity by echocardiography is highly recommended. (8)

Transesophageal echocardiography and three-dimensional, real-time echocardiography provide a more accurate evaluation of the LV aneurysm morphological features, detecting the eventual thrombosis and accurately quantifying the severity of mitral regurgitation.

Cardiac magnetic resonance imaging (CMR) emerged as a novel imaging modailty in the evaluation of LV aneurysm. Due to tissue characterization, CMR is able to distinguish the signal of the pericardium and myocardium, differentiating true inferior LV aneurysms from pseudoaneurysm (9).

Therapeutic strategy for inferobasal LV aneurysm is usually conservative because the risk of rupture is low. Guidelines recommend angiotensin-converting enzyme inhibitors or angiotensin receptor blockers and aldosterone antagonist to reduce negative LV remodeling and improve survival; the finding of a mural thrombus requires anticoagulation.(10)

Surgical treatment is carried out in patients presenting with refractory clinical manifestations such as heart failure, angina, arrhythmia and embolism.

The goal of surgical intervention is to correct the size and geometry of the left ventricle, reduce wall tension and paradoxical movement, and improve systolic function.(11)
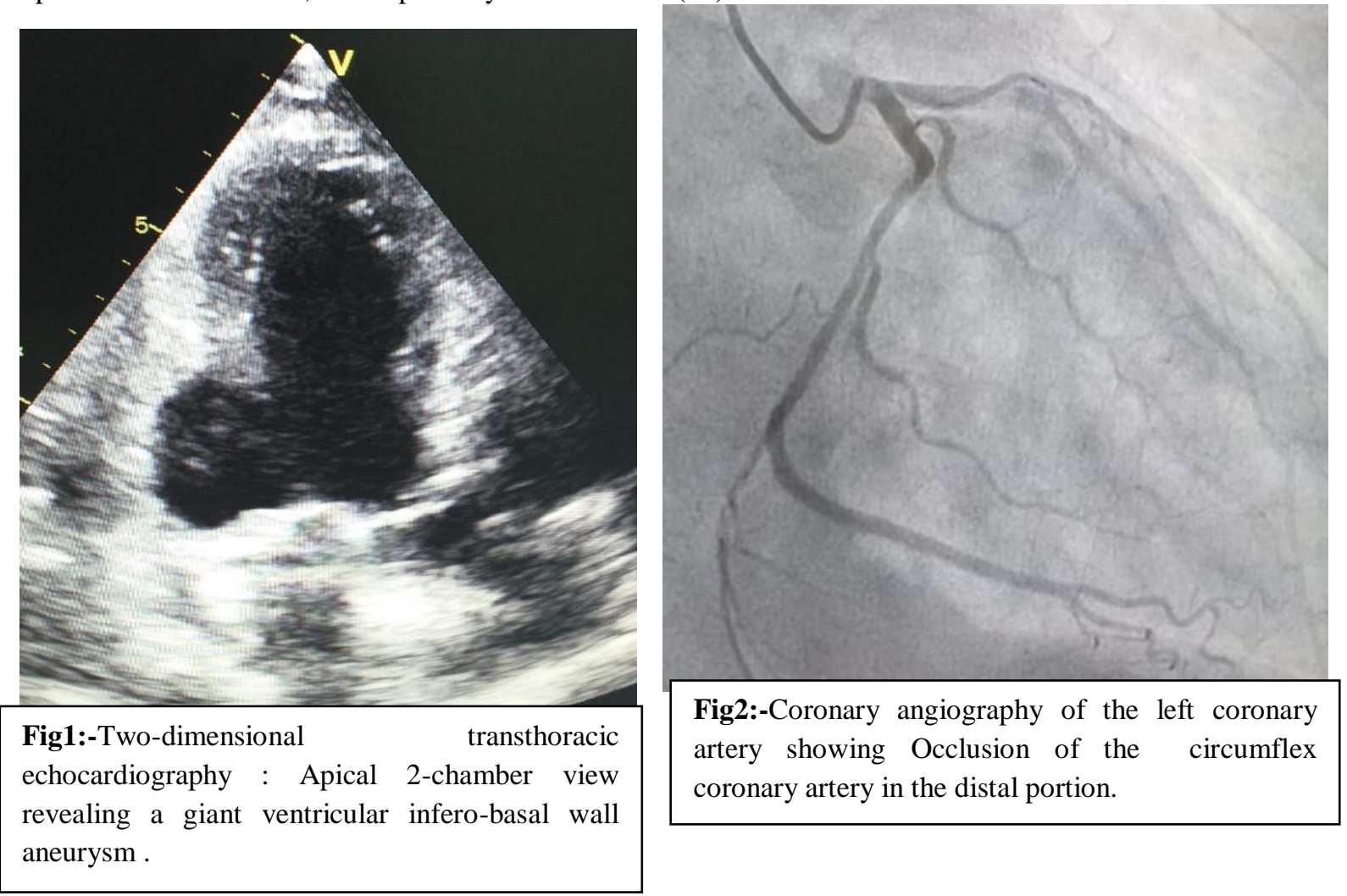
The aneurysms appear like a thin, akinetic or dyskineticregion and collapse inward when the ventricle is vented duringsurgeryThe aneurysms appear like a thin, akinetic or dyskineticregion and collapse inward when the ventricle is vented during surgery.

\section{References:-}

1. Weisman HF, Healy B. Myocardial infarct expansion, infarct extension, and reinfarction: pathophysiologic concepts. Prog Cardiovasc Dis 1987;30:73-110.

2. Zofolli G,Mangino D, Venturini A, et al. Diagnosing left ventricular aneurysm from pseudo-aneurysm: a case report and a review in literature. J Cardiothorac Surg 2009;4:11.

3. Tikiz H, Balbay Y, et al. The effect of thrombolytic therapy on left ventricular aneurysm formation in acute myocardial infarction: relationship to successful reperfusion and vessel patency. Clin Cardiol 2001;24(10):65662

4. Vargas SO, Sampson BA, Schoen FJ. Pathologic detection of early myocardial infarction: a critical review of the evolution and usefulness of modern techniques. Mod Pathol 1999;12:635-45.

5. Paul M, Schafers M, Grude M, et al. Idiopathic left ventricular aneurysm and sudden cardiac death in young adults. Europace 2006;8:607-12.

6. Loperfido F, Pennestrì $F$, et al. Diagnosis of left ventricular pseudoaneurysm by pulsed Doppler echocardiography. Am Heart J 1985;110(6):1291-3.

7. Roelandt JR, Sutherland GR, et al. Improved diagnosis and characterization of left ventricular pseudoaneurysm by Doppler color flow imaging. J Am Coll Cardiol 1988;12(3):807-11.

8. Singh S. Large basal inferior wall aneurysm with thrombus: a rare phenomenon revisited. BMJ Case Rep 2012.

9. Cho MN, Mehta SK, et al. Differentiating true versus pseudo left ventricular aneurysm: a case report and review of diagnostic strategies. Cardiol Rev 2006;14(6):e27-30.

10. Task Force on the management of ST-segment elevation acute myocardial infarction of the European Society of Cardiology (ESC), Steg PG, et al. ESC Guidelines for the management of acute myocardial infarction in patients presenting with ST-segment elevation. Eur Heart J 2012;33(20):2569-619.

11. Lundblad R, Abdelnoor M, et al. Surgery for left ventricular aneurysm: early and late survival after simple linear repair and endoventricular patch plasty. J Thorac Cardiovasc Surg 2004;128(3):449-56. 